\title{
Chronic active hepatitis: a sixteen year survey at a district general hospital
}

\author{
A.R. Tanner and A.W. Dellipiani \\ Department of Medicine, North Tees General Hospital, Hardwick Estate, Hardwick Estate, Stockton on Tees, \\ Cleveland TS19 8PE, UK
}

\begin{abstract}
Summary: Over a period of 16 years (1971 -87) all cases of chronic active hepatitis (CAH) diagnosed following liver biopsy have been reviewed to assess incidence, aetiology, outcome and response to treatment. North Tees District serves a population of 210,000 and 26 cases were identified (20 female) age range 9-73 years (median 56 years). Incidence remained constant at 1:100,000/year. Twenty cases (77\%) had an immune aetiology. Other aetiologies were hepatitis B virus (HBV) infection, alpha-1AT deficiency, non-A non-B hepatitis and a complex multisystem disease. The median follow-up period was $\mathbf{5 0}$ months.

There were 7 deaths, three unrelated to liver disease. Survival analysis gives an 86\% 5-year survival and $56 \%$ 10-year survival. Twenty four patients were treated with steroids (and 6 additionally with azathioprine); $15(63 \%)$ were steroid responsive and 9 were non-responsive. In five patients steroids were successfully discontinued but in 10 patients severe symptomatic relapse occurred on steroid reduction below $7.5-10 \mathrm{mg} / \mathrm{day}$. Steroid non-responders were not typical CAH, 5 with predominantly a rise in alkaline phosphatase, one multisystem disease and one HBV positive. Steroid discontinuation was only possible in one third of the patients responding. Eighteen subjects (69\%) were cirrhotic at initial biopsy; 9 had follow-up biopsies, 6 were unchanged but three had progressed to cirrhosis despite apparent steroid responsiveness.
\end{abstract}

\section{Introduction}

Published studies on chronic active hepatitis (CAH) have come from large referral centre. ${ }^{1-5}$ Although the crucial studies at the start of the last decade defined the condition and established appropriate treatment regimes, certain aspects of the condition, as seen in a non-referral centre, have not been emphasized despite increasing knowledge of the pathogenesis of $\mathrm{CAH}^{.5-8}$ The present study establishes the incidence of the condition in a defined population, assesses changes in the pattern of inci' ence, measures long-term prognosis and treant outcome, and categorizes the aetiolory in iis non-referral population.

\section{Methods}

A computer search was made for all diagnostic categories which might include cases of $\mathrm{CAH}$ using the Hospital Activity Analysis database for the period 1971-1987 for a single health district (North Tees). The North Tees Health District serves a population of 210,000 and this has remained reasonably stable

Correspondence: A.R. Tanner, D.M., F.R.A.C.P.

Accepted: 26 May 1989 throughout the time of the study. The initial search produced 405 case records. A secondary search of pathology records was also made. All appropriate case records were reviewed and any subjects with a history of alcohol abuse and histological findings of alcoholic liver disease were rejected. No patients with a history of both alcohol abuse and histological findings of $\mathrm{CAH}$ were identified. Only the records of those subjects with chronic active hepatitis established histologically were subsequently analysed. All histology was reviewed blind by a single observer (ART). The original sections were retrieved and coded to ensure that patient identification was not possible. In all subjects transaminase measurements in the first 3 months of the illness exceeded $200 \mathrm{IU}$ on at least one occasion and all subjects undergoing liver biopsy were experiencing specific hepatic or non-specific constitutional symptoms at the time of biopsy. Histologically, $\mathrm{CAH}$ was defined by the presence of piecemeal necrosis with a variable degree of lobular involvement.

During the period of time 1971-1985 a single gastroenterologist (AWD) was involved in the care of these patients either directly or by consultation; from 1985-1987 both authors have been involved. To our knowledge, no cases have been referred outside the 
district for diagnosis and treatment during this period. Since a single gastroenterologist has worked in this District, which has a single hospital, for all of this period and a policy of close cooperation between the consultants for the management of specialist cases is well established, it is unlikely that any cases have been missed or wrongly categorized. Local ethical committee approval was obtained for this case-note review.

Kaplan-Meier analysis was used to establish survival.

\section{Results}

Twenty-six cases of CAH have been identified (20 female), all Caucasian. The median age of onset was 56 years (range 9-73 years); the age distribution is shown in Figure 1. The incidence of CAH is approximately $1: 100,000 /$ year and has remained unchanged over this time period (6-7 cases in the District every 5 years). In all except 6 subjects presentation was with an acute hepatitic illness and initial transaminase measurement was $>500 \mathrm{IU} / 1$ in 19 subjects. In most

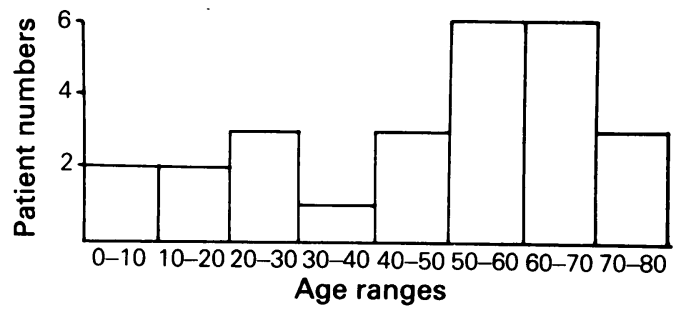

Figure 1 Age distribution at presentation of subjects with chronic active hepatitis (North Tees Health District, 1971-1987). patients a prodrome of malaise and weight loss over az number of months preceded the presentation at hos $\frac{\bar{D}}{2}$ pital.

Investigations relating to aetiology are shown in Table I and the presumed aetiology in this group of ? patients is shown in Table II. All 26 subjects were screened for HBV infection (hepatitis B surface antigen and core antibody); 11 subjects (all aged? $<50$ years) have been assessed for Wilson's disease. $\varrho$ Alpha-1-antitrypsin testing (including phenotyping)en has been done in 10 subjects and autoantibodies in $25-$ subjects. Fourteen subjects $(56 \%)$ had a positiveanti-nuclear factor (ANF), 5 subjects $(20 \%)$ positive $\vec{\omega}$ smooth muscle antibody and one (4\%) antimitochondrial antibody (AMA). In 12 subjects IgGo levels were markedly raised $(>18 \mathrm{~g} / \mathrm{l})$. IgM and $\operatorname{IgA}$. levels were raised in 9 and 5 subjects respectively. $/ 5$ Primary biliary cirrhosis (PBC) variant was defined as those patients with predominant cholestasiseo biochemically who had either a positive AMA (oneN patient) or a strikingly elevated IgM level (two patients). The histological pattern was more in favour $\supset$ of $\mathrm{CAH}$.

Clinical management was guided largely by symptomatology and transaminase measurement. Repeat biopsies were performed in subjects where adequate control was not achieved within 1-2 years or whgn $\overrightarrow{0}$ subsequent relapse proved difficult to control. Eigू $-\infty$ teen patients $(69 \%)$ were cirrhotic at initial biopey. Nine had follow-up biopsies and of these, 6 wereo unchanged ( 5 cirrhotic; 1 non-cirrhotic) and 3 had progressed to cirrhosis despite steroid responsiveness. All but one of the follow-up biopsies showed $a \stackrel{\circ}{\circ}$ diminution in inflammatory activity.

Twenty four subjects were treated with steroids $\overrightarrow{\vec{O}}$ (30-60 mg prednisolone daily) and, of these, 15 were 3 steroid responsive using clinical and biochemical criteria, although 6 required the addition of azathio-

Table I Investigations performed and results in 26 cases of chronic active hepatitis

\begin{tabular}{lccc}
\hline Investigation & Number tested & Number positive \\
\hline HBV* markers & 26 & & 2 \\
Copper studies & 11 & & $0 \dagger$ \\
Alpha-1-antitrypsin studies & 10 & ANF & 14 \\
Autoantibodies & 25 & SMA & 5 \\
& & AMA & 1 \\
& & Raised IgG & 12 \\
Immunoglobulins & 21 & Raised IgM & 9 \\
& & Raised IgA & 5 \\
\hline
\end{tabular}

* HBsAg and HBcAb; †one subject heterozygous for Wilson's disease; $\ddagger$ both MS phenotype.

ANF - antinuclear factor; SMA - smooth muscle antibody; AMA - antimitochondrial antibody. 
Table II Aetiology of chronic active hepatitis

\begin{tabular}{lrc}
\hline & Female & Male \\
\hline Autoimmune & 10 & 1 \\
$\quad$ Definite* & 4 & \\
Probable* & 3 & \\
PBC variant $\dagger$ & 1 & 2 \\
HBV infection & 1 & \\
Alpha 1 AT deficiency & 1 & \\
Possible NANB infection $\ddagger$ & & 1 \\
Multisystem immune disease & & 1 \\
\hline Systemic lupus erythematosus & & \\
Sclerosing cholangitis &
\end{tabular}

*'Definite' defined as those patients with autoantibodies and IgG $>20 \mathrm{~g} / \mathrm{l}$ and/or splenomegaly; 'Probable' defined as those patients without autoantibodies but $\operatorname{IgG}>20 \mathrm{~g} / \mathrm{l}$, and rapid resolution with steroid administration.

+ PBC primary biliary cirrhosis - see text.

‡No autoimmune markers detected, immunoglobulins normal, hepatitis markers negative, indolent course with no response to steroids.

prine to maintain symptomatic and biochemical remission at a reasonable dose level of prednisolone $(<10 \mathrm{mg})$. Of those not responding to steroids, atypical features (for classical 'lupoid hepatitis') were common; one subject was HBV positive, one had a poorly defined multisystem disease (coeliac, hypothyroid, fibrosing alveolitis), and 5 predominantly a rise in alkaline phosphatase [alpha-1AT deficiency 1, PBC variant 3, probable non A non B (NANB) hepatitis 1]. Of these 'non-responders', three $(33 \%)$ died of hepatic complications (median followup, 96 months) compared to one $(7 \%)$ of 15 'responders' (median follow-up, 48 months). Steroid withdrawal was attempted in all 15 steroid responsive patients but 10 subjects experienced severe symptomatic relapses on reduction of steroid dosage to between $7.5-10 \mathrm{mg} /$ day. In two of these relapsing patients, initially steroid responsive, control was not reestablished. In 5 subjects steroids were withdrawn successfully at between 2 and 3 years from initial treatment and followed up for a mean of 36 months.

The median follow-up period was 50 months (48 months for survivors, 71 months for subjects who died) and survival analysis (Figure 2) shows an $86 \%$ 5 -year survival, 56\% 10-year survival. There were 7 deaths, 3 unrelated to liver disease (myocardial infarct, peripheral vascular disease, respiratory failure) and 4 directly related to the liver disease (bleeding varices 2 , hepatic encephalopathy 2).

\section{Discussion}

Chronic active hepatitis is a histological diagnosis supported by various clinical and immunological

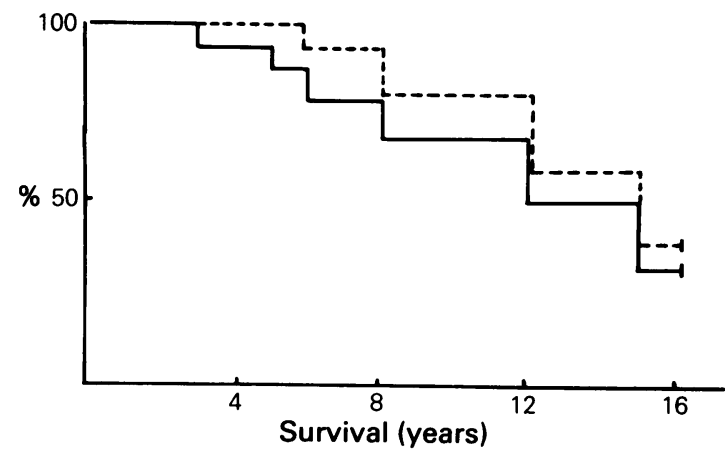

Figure 2 Survival curves for patients with chronic active hepatitis, Kaplan-Meier analysis. Interrupted line indicates survival curve censored for non-hepatic deaths.

features, the aetiology being quite variable. Even in this study a wide spectrum of aetiologies is represented, apart from drug-related $\mathrm{CAH}$. Drugs that have commonly been associated with CAH include oxyphenisatin, alpha-methyl-dopa, nitrofurantoin and isoniazid. ${ }^{8}$ Although classic autoimmune $\mathrm{CAH}$ is described predominantly in females in the second and third decades and is associated with a high prevalence of various autoantibodies, these patients are now commonly seen in a non-referral centre. As shown in the present study, the age of onset is predominantly in late middle age, commonly with an immune aetiology (77\%) but with a much lower prevalence of autoantibodies than is seen in 'lupoid' CAH. As in previous studies, ${ }^{9,10}$ a small proportion (12\%) of cases are difficult to classify, with clinical and biochemical features suggestive of PBC but histology consistent with $\mathrm{CAH}$. These features did not predict response to steroid therapy.

Despite observations from referral centres suggesting a fall in incidence, new case presentations have remained quite stable in the North Tees District over the last 16 years. The decline in numbers at the centres probably reflects the greater confidence in managing this condition in non-referral institutions. A high proportion of patients had established cirrhosis $(69 \%)$ at initial presentation. This compares with a figure of $31 \%$ for the Mayo Clinic in their group $(n=180)$ of severe hepatitis B surface antigen negative $\mathrm{CAH} .{ }^{11}$

Despite the older age range of the subjects the 10 -year survival in the present study $(56 \%)$ is very similar to the treatment group reported from the Royal Free Hospital (63\%). ${ }^{12}$ As might be expected, death was not necessarily attributable to the underlying liver disease. However, one of the patients died following discontinuation of steroids and a subsequent severe steroid unresponsive relapse. A study from King's College Hospital ${ }^{13}$ has shown that in 
CAH subjects with detectable serum autoantibodies and a severe histological appearance a very high rate of relapse $(87 \%)$ can be expected on attempting treatment withdrawal. Seven of the 10 relapsing patients in the present study showed prominent autoantibodies but all 5 who have withdrawn successfully from steroids also demonstrated high titre autoantibodies. In practical terms, autoantibodies did not seem to be a good guide to those in whom steroid withdrawal will be safely and successfully achieved. Unfortunately, anti-liver-specific lipoprotein (perhaps a better indicator of steroid requirement $)^{14}$ has not been measured in the present study.

\section{References}

1. Cook, G.C., Mulligan, R. \& Sherlock, S. Controlled prospective trial of corticosteroid therapy in active chronic hepatitis. $Q J$ Med 1971, 40: 159-185.

2. Murray-Lyon, I.M., Stern, R.B. \& Williams, R. Controlled trial of prednisone and azathioprine in active chronic hepatitis. Lancet 1973, i: 735-737.

3. Soloway, R.D., Summerskill, W.H.J., Baggenstoss, A.H. et al. Clinical, biochemical, and histological remission of severe chronic active liver disease: a controlled study of treatments and early prognosis. Gastroenterology 1972, 63: $820-833$.

4. Lam, K.C., Lai, C.L., Ng, R.P., Trepo, C. \& Wu, P.C. Deleterious effect of prednisolone in HBsAg-positive chronic active hepatitis. $N$ Engl J Med 1981, 304: $380-386$.

5. Hodges, J.R. Millward-Sadler, G.H. \& Wright, R. Chronic active hepatitis. The spectrum of disease. Lancet 1982, i: $550-552$.

6. Schalm, S.W., Summerskill, W.H.J., Gitnick, G.L. \& Elveback, L.R. Contrasting features and responses to treatment of severe chronic active liver disease with and without hepatitis Bs antigen. Gut 1976, 17: 781-786.

7. Koretz, R.L., Stone, O. \& Gitnick, G.L. The long-term course of non-A, non-B post transfusion hepatitis. Gastroenterology 1980, 79: 893-898.

8. Maddrey, W.L. \& Boitnott, J.K. Drug-induced chronic liver disease. Gastroenterology 1977, 72: 1348-1353.
This study has described the pattern of $\mathrm{CAH}$ in a defined population. The incidence of the condition (1:100,000/year) can be definitely stated and has not changed in recent years. Treatment outcome $(86 \%$ survival at 5 years) is very similar to results from major referral centres and the difficulties with steroid withdrawal are again emphasized. Deaths from hepatic complications tend to occur late in the course of the illness, the survival curves showing a steady decline with time. In contrast to textbook descriptions, subjects tend to be middle aged with a relatively low prevalence of autoantibodies and $69 \%$ have established cirrhosis at initial presentation.

9. Shouval, D., Levij, I.S. \& Eliakim, M. Chronic active hepatitis with cholestatic features. Am J Gastroenterol 1979, 72: 542-550.

10. Geubel, A.P., Baggenstoss, A.H. \& Summerskill, W.H.J. Responses to treatment can differentiate chronic active liver disease with cholangitic features from the primary biliary cirrhosis syndrome. Gastroenterology 1976, 71: 444-449.

11. Baggenstoss, A.H., Soloway, R.D., Summerskill W.H.J., Elveback, L.R. \& Schoenfield, L.J. Chronic active liver disease. The range of histologic lesions, theio response to treatment, and evolution. Hum Pathol 1972 3: $183-198$.

12. Kirk, A.P., Jain, S., Pocock, S., Thomas, H.C. \& Sherlock, S. Late results of the Royal Free Hospital controlled trial of prednisolone therapy in hepatitis B surface antigen negative chronic active hepatitis. Gut 1980, 21: 78-83.

13. Hegarty, J.E., Nouri-Atria, K.T., Portman, B., Eddleston, A.L.W.F. \& Williams, R. Relapse following treatment withdrawal in patients with autoimmune chronic active hepatitis. Hepatology 1983, 3: 685-689.

14. McFarlane, I.G., Hegarty, J.E., McSorley, C.G., McFarlane, B.M. \& Williams, R. Antibodies to liver specific protein predict outcome of treatment withdrawal in autoimmune chronic active hepatitis. Lancet 1984, ii: 954-956. 